United Nations Educational Scientific and Cultural Organization and

International Atomic Energy Agency

THE ABDUS SALAM INTERNATIONAL CENTRE FOR THEORETICAL PHYSICS

\title{
MULTIOBJECTIVE BAK-SNEPPEN MODEL ON A SMALL-WORLD NETWORK
}

\author{
M. F. Elettreby ${ }^{1}$ \\ Mathematics Department, Faculty of Science, Mansoura University, \\ Mansoura 35516, Egypt \\ and
}

The Abdus Salam International Centre for Theoretical Physics, Trieste, Italy.

\begin{abstract}
Small-world networks (SWN) are relevant to biological systems. We study the dynamics of the Bak-Sneppen (BS) model on small-world network, including the concepts of extremal dynamics, multiobjective optimization and coherent noise. We find that the small-world structure stabilizes the system. Also, it is more realistic to augment the Bak-Sneppen model by these concepts.
\end{abstract}

MIRAMARE - TRIESTE

September 2004

${ }^{1}$ mohfathy@mans.edu.eg 


\section{Introduction}

Networks have been used to model the evolutionary dynamics of biological and many other systems [1]. There are two important measures to any network.

Definition 1 The characteristic path length $L$ is the measure of the typical separation between any two vertices in the network.

The value of the characteristic path length $L$ is equal to the number of edges in the shortest path connect by any two vertices, averaged over all pairs of vertices [2].

Definition 2 The clustering coefficient $C$ measures the cliquishness of a typical neighborhood. It is the average fraction of pairs of neighbors of a vertex which are also neighbors of each other.

Suppose that a vertex $v$ has $k_{v}$ neighbors, then there are at most $\frac{k_{v}\left(k_{v}-1\right)}{2}$ edges between them. Let $C_{v}$ denote the fraction of the edges that actually exist, then the value of $C$ is the average of all $C_{v}$ 's. The characteristic path length $L$ is a global property whereas the clustering coefficient $C$ is a local property of the network [3].

Generally, there are two kinds of networks, the regular and the random networks.

Definition 3 The regular network consists of $N$ vertices arranged regularly on a lattice such that each vertex has the same number $N N$ of nearest neighbors, this number is known as the coordination number.

Regular networks have a highly clustered and large world-effect (large characteristic path length $L$ ), where $L$ grows linearly with $N$ in these networks.

Definition 4 The random network consists of $\frac{N \mathrm{Z}}{2}$ edges that connect $N$ vertices randomly, where $\mathbf{Z}$ is the average number of neighbors to all sites in the network.

Random networks have poorly clustered as well as small-world effect, where $L$ grows with $\log (N)$. Large values of $C$ are usually associated with large values of $L$ and vice versa [4].

The real networks are somewhere between these two kinds of networks. The majority of dynamical systems were described by completely regular or completely random networks. Watts and Strogatz [5] suggest a simple model of networks by rewiring the edges with probability $p$, $0 \leq p \leq 1$, of a regular network to produce an increasing amount of disorder. We can get the regular network by putting $p=0$, whereas if $p=1$, we get the random network. For small values of $p$, each shortcut has a highly nonlinear effect on $L$. It contracts the distance not only between the pair of vertices that it connects, but also between their immediate neighbors and hence the neighbors of their neighbors and so on. By contrast, the removed edge from a clustered neighborhood has a linear effect on $C$. Although, the number of nearest neighbors of any particular vertex in the new graph can be greater or smaller than the coordination number 
$\mathbf{N N}$, the new graph still has $N$ vertices, an average number of nearest neighbors equal to $\mathbf{Z}$ and some shortcuts.

Definition 5 The small-world network (SWN) has the property of highly clustered and a small-world effect.

This new class of networks can be used to describe a wide range of real systems [6].

The evolutionary history of most species is characterized by long periods of stasis punctuated by relatively brief intervals of rapid evolutionary activity [7]. Self-organized criticality (SOC) refers to the tendency of large dynamical systems to organize themselves into a steady state, far out of equilibrium with propagating avalanches of activity of all sizes [8].

The most common interactions among species in this ecosystem are predation, competition and mutualism. As a result of these interactions the evolutionary adaptation of one species must affect on its nearest neighbors. Also, these interactions can lead to large evolutionary disturbances, termed co-evolutionary avalanches. Each species in these evolution models are characterized by only one fitness.

Bak and Sneppen [9], proposed a self-organized model known as the BS model to explain the punctuated equilibrium of biological evolution. They considered a one-dimensional model of an ecosystem consisting of $N$ species with periodic boundary conditions, topologically a circle. They assign a fitness value $0<f(i)<1$ to each species $i, i=1,2, \ldots, N$. At each time-step, the species $j$ with the minimum fitness $f(j)$, is sought, and then it replaced, together with its nearest neighbors $j \pm 1$, by new ones that are randomly distributed. After running the system for a sufficiently long time most of the fitness are greater than the certain threshold value $(0.667 \pm 0.001)$. Also, the distribution of the distance $d$ between subsequent mutations as well as the distribution of avalanches sizes $s$ exhibit power law,

$$
\begin{aligned}
& P(d) \sim d^{-\tau_{d}}, \\
& P(s) \sim s^{-\tau_{s}} .
\end{aligned}
$$

The idea of coherent noise [10] has been proposed to interpret the problem of mass extinction where species die out as a result of environmental stress $\eta$ such as the effect of earthquakes, change in the level sea, etc. The species under environmental stress will survive if their fitness $>\eta$.

Grassberger [11] suggests an evolution model depending on the environmental stress. He gets a power law distribution of the avalanche size (1) with $\left(\tau_{s} \simeq 2\right)$ even without species interactions. Ahmed, et al. [12] argued that the two ideas, SOC and coherent noise, are not contradictory but rather complementary to each other, since species interactions and environmental effects should be included. They get a power law distribution of the avalanche size with $\left(\tau_{s} \simeq 2.09\right)$. 


\section{The Bak-Sneppen model on a small-world network}

Models of dynamical systems with SWN display enhanced signal propagation speed, computational power and synchronizability [3]. SWN are crucially important in the case of communications, the spread of diseases, etc. So, it is more real and efficient to study the BS model on SWN with the coherent noise concept.

There are many different models that describe the biological evolution and extinction. The important point in these models $[10,13,14,15]$ is that the distribution of the sizes of avalanches is a power law, although they get different values of the exponent $\tau_{s}$. Some of these models predict a power law distribution for other quantities such as life time of species, the distance between subsequent mutations, etc., such as the BS model.

Now, we will study the first modification of the BS model namely the BS model on a SWN. We assign a fitness value $0<f(i)<1$ to each species $i, i=1,2, \ldots, N$, where $N$ is the number of species in the ecosystem with periodic boundaries. Define the edges with values $E(i, j)$ which connect any species $i$ with its nearest neighbors $j^{\prime} s$, such that,

$$
0<E(i, j)<1, \quad E(i, j)=E(j, i), \quad \forall i, j=1,2, \ldots, N
$$

Let $f(m)$ be the minimum fitness among all species, then change the fitness value $f(m)$ and its neighbors. Now, we rewire the edges as follows if: $E(i, j)<p$, then cut this edge and reconnect the site $i$ with a site $k$ where $k \neq i, j$ and $1 \leq k \leq N$.

First, we take the number of nearest neighbors $N N=2$ (as the original BS model) and $p=0.00$ (regular network) then run the system with $N=4096$ species for $10^{7}$ time-step. We get Fig. 1 which is similar to the figures in [9].

Secondly, at $p=0.04$ we get Fig.2, where all fitness values are above the threshold value $(0.62 \pm 0.01)$. Also, the distribution of the distance between subsequent mutations as well as the distribution of the avalanche size exhibit power law.

Finally, we introduce the coherent noise effect by assuming that if $f(i) \leq \eta$, where $\eta$ is the environmental effect which is chosen randomly at each time-step, then change $f(i)$ and its neighbors. In Fig.3, we run the system with $N=1000$ species for $2 \times 10^{6}$ time-step, and hence we find that all fitness are above the threshold value $(0.31 \pm 0.01)$.

\section{Random Bak-Sneppen model on a small-world network}

In real biological systems the lowest fitness and some of the low fitness species are updated, this phenomenon is known as extremal dynamics $[16,17]$. In this case the number of updated fitness is a random number.

So, we will study this random version of BS model on a SWN. At each time-step, a uniformly distributed random number is picked up and all the species with fitness value less than this number will update. This extremal dynamics has been used to explain the long term memory 
for the immune system [17] and to solve some optimization problems such as spin glass, graph coloring and graph partitioning $[18,19]$. When we run a system with $N=4096$ species for a different sufficiently long time (up to $2 \times 10^{7}$ ), we find that most of the fitness are above certain threshold value 0.64 [20].

Instead of running these rules on a regular graph, we will run the system on a SWN with $N=1000$ species at $p=0.02,0.04,0.06$ and $N N=2,4,6$ (nearest neighbor) for different iterations $t=1 \times 10^{5}, 1 \times 10^{6}, 1 \times 10^{7}$. In Fig.4, we find that the system reaches a dynamical steady state more quickly than the regular case and most of the fitness are above certain threshold value $(0.25 \pm 0.01)$. Also, the distribution of avalanches still have a power law form. These results imply that small-world network structure stabilizes the system.

\section{Multiobjective optimization model on a small-world network}

In biology, most of real life optimization problems are multiobjective ones (MOB), such as objective of foraging and of minimizing predation risk [21]. Most evolution models consider only one fitness i.e. single objective optimization. Therefore, it is important to generalize the standard single goal oligopoly studies to multiobjective ones. The methods of MOB optimization are intuitive.

The first method is known as the lexicographic method. In this method, objectives are ordered according to their importance. Then the first objective is satisfied fully. The second one is satisfied as much as possible given that the first objective has already been satisfied and so on. One of the famous applications is the university admittance where students with the highest grades take position in the college which they prefer. The second objective will take position from the remaining places and so on. This method is useful but in some cases it is not applicable.

The second method is the weights method [22]. Assume that it is required to minimize the objectives $Z(i), i=1,2, \ldots, N$. Define the following quantity:

$$
Z=\sum_{i=1}^{N} w(i) Z(i),
$$

where

$$
w(i) \geq 0 \quad \text { and } \quad \sum_{i=1}^{N} w(i)=1 .
$$

Then the problem becomes minimization of the quantity $Z$. We can easily implement this method but there are some drawbacks. The first drawback, it may give a Pareto dominated solution.

Definition 6 A solution $Z^{\prime}(i), \quad i=1,2, \ldots, N$ is called a Pareto dominated solution if there is another solution $Z(i), \quad i=1,2, \ldots, N$ such that $Z(i) \leq Z^{\prime}(i)$ for all $i$ with at least one $k$ such that $Z(k)<Z^{\prime}(k)$. 
The second drawback, it is difficult to apply this method for large $N$.

In the third method, we minimize only one objective while we set the other objectives as constraints e.g. minimize $Z(1)$ subject to $Z(i) \leq a(i), \quad i=2,3, \ldots, N$ where $a(i)$ are parameters to be updated. The problem in this method is the choice of the thresholds $a(i)$. In the case of equality i.e. $Z(i)=a(i)$ this method gives a Pareto optimal solution.

The fourth method uses fuzzy logic to study each objective individually and determines its maximum $Z_{\max }(i)$ and its minimum $Z_{\min }(i)$. Then determine a membership for each $i$,

$$
m(i)=\frac{Z_{\max }(i)-Z(i)}{Z_{\max }(i)-Z_{\min }(i)}, \quad i=1,2, \ldots, N,
$$

where $0 \leq m(i) \leq 1$, then find $\max \{\min (m(i), i=1,2, \ldots, N)\}$. This method is a bit difficult to apply for a large number of objectives, also, it gives a Pareto optimal solution.

The BS model is generalized to the multiobjective one by assigning two fitness $f_{1}(i), f_{2}(i)$ to each species instead of one fitness [20]. In this case, if the fitness,

$$
x(i)=\alpha f_{1}(i)+(1-\alpha) f_{2}(i), \quad 0<\alpha<1
$$

is less than the minimum fitness, then update each of $f_{1}(i), f_{2}(i)$ and their nearest neighbors $f_{1}(i \pm 1), f_{2}(i \pm 1)$. In the updating rule, we use the weights method in MOB.

Now, we use the same rules on a SWN by choosing arbitrary values, $p=0.04, N N=2$ at $\alpha=0.3,0.5,0.9$. Then run a system with $N=4096$ species for a different sufficiently long time (up to $5 \times 10^{7}$ ). Figure 5 shows that the system reaches a dynamical steady state more quickly than the regular case and most of the fitness are above a certain threshold value $(0.59 \pm 0.01)$. Also, the distribution of the avalanche sizes still has a power law form. Finally, if $x(i) \leq \eta$ for each species $i$, where $\eta$ is the environmental effect, then change $x(i)$ and its nearest neighbors (i.e. coherent noise effect). When, we run a system with $N=1000, p=0.04, N N=2$ and $\alpha=0.9$ for $5 \times 10^{6}$ time-step, where $\eta$ changes at each time-step, then all fitness are above the threshold value (0.3), see Fig.6. These results imply that SWN structure stabilizes the system. Also, it is important to augment the BS model by the coherent noise as well as by the multiobjective optimization.

\section{Acknowledgments.}

The author, a Junior Associate of the ICTP, would like to thank the Abdus Salam International Centre for Theoretical Physics (ICTP), Trieste, Italy, for financial support and facilities. He would also like to thank Prof. E. Ahmed for useful discussions and comments. 


\section{References}

[1] L. Sattenspiel and C. P. Simon. The spread and presistance of infectious disease in structured populations. Math. Biosci., 1988;90: 369-366.

[2] S. Milgram. The small-world problem. Psychology Today, 1967;2: 60-67.

[3] M. E. J. Newman. Models of the Small World. J. Stat. Phys., 2000;101: 819-841.

[4] B. Bollobas. Random Graphs. New York: Academic Press, 1985.

[5] D. J. Watts and S. H. Strogatz. Collective dynamics of small-world networks. Nature, 1998;393: 440-442.

[6] D. J. Watts. Small Worlds. Princeton University Press, 1999.

[7] S. Gould and N. Eldredge. Punctuated equilibrium comes of age. Nature, 1993;366: 223-227.

[8] P. Bak. How nature works: the science of self-organized criticality. NewYork: SpringerVerlag, 1996.

[9] P. Bak and K. Sneppen. Punctuated equilibrium and criticality in a simple model of evolution. Phys. Rev. Lett., 1993;71: 4083-4086.

[10] M. E. J. Newman, A model of mass extinction. J. Theor. Biol., 1997;189: 235-252.

[11] P. Grassberger. Are damage spreading transitions generically in the universality class of directed percolation. J. Stat. Phys., 1995;79: 13-23.

[12] E. Ahmed, M. F. Elettreby and H. N. Agiza. Comments on some extinction models. Int. J. Mod. Phys. C, 20001;3: 615-618.

[13] C. Adami. Self-organized criticality in living systems. Phys. Lett. A, 1995;203: 29-32.

[14] P. Bak, C. Tang and K. Wiesenfeld. Self-organized criticality: An explanation of the 1/f noise. Phys. Rev. Lett., 1987;59: 381-384.

[15] K. Sneppen, P. Bak, H. Flyvbjerg and M. H. Jansen. Evolution as a self-organized critical phenomenon. Proc. Nat. acad. Sci., 1995;92: 5209-5213.

[16] D. Head. Extremal driving as a mechanism for generating long-term memory. J. Phys. A, 2000;33: $387-391$.

[17] E. Ahmed and A. H. Hashish. On modeling of immune memory mechanisms. Theor. BioSci., 2003;122: 349-352.

[18] S. Boettcher and A. Percus. Optimization with extremal dynamics. Phys. Rev. Lett., $2001 ; 86 ; 5211-5214$.

[19] E. Ahmed and M. El-Alem. Immune-motivated optimization. Int. J. Theor. Phys., 2002;41: 985-990.

[20] E. Ahmed and M. F. Elettreby. On multiobjective evolution model. Int. J. Mod. Phys. C (to be appear).

[21] M. Zeleny. Multiple Criteria Decision Making. NewYork: McGraw Hill, 1982.

[22] L. Zadeh. Optimality and non-scalar-valued performance criteria. IEEE Transactions on Automatic Control, 1963;8: 59-60. 


\section{Figure Captions}

Figure 1 The results of the BS model under our simulations in a system of size $N=4096, p=$ 0.00 , the coordination number $N N=2$ and $t=10^{7}$ iteration. (a) Distribution $P(F)$ of the fitness $F$ in the critical state (right curve) with the distribution of minimum fitness (left curve). (b) The distribution $P(D)$ of the distances $D$ between subsequent mutations in log-log plot. (c) Punctuated equilibrium behavior, activity vs time in a local segment of twenty consecutive species. (d) Distribution $P(S)$ of the avalanche size $S$ in the critical state in a log-log plot.

Figure 2 The results of the BS model on SWN in a system of size $N=4096$ species, $t=10^{7}$ iteration, $p=0.04$ and $N N=2$. (a) Distribution $P(F)$ of the fitness $F$ in the critical state (right curve) with the distribution of minimum fitness (left curve). (b) The distribution $P(D)$ of the distances $D$ between subsequent mutations in $\log$-log plot. (c) Punctuated equilibrium behavior, activity vs time in a local segment of twenty consecutive species. (d) Distribution $P(S)$ of the avalanche size $S$ in the critical state in a log-log plot.

Figure 3 The results of the BS model on SWN with the coherent noise in a system of size $N=1000$ species, $10^{7}$ iteration, $p=0.04$ and $N N=2$. (a) Distribution $P(F)$ of the fitness $F$ in the critical state (right curve) with the distribution of minimum fitness (left curve). (b) The distribution $P(D)$ of the distances $D$ between subsequent mutations in $\log$-log plot. (c) Punctuated equilibrium behavior, activity vs time in a local segment of twenty consecutive species. (d) Distribution $P(S)$ of the avalanche size $S$ in the critical state in a log-log plot.

Figure 4 A system of size $N=1000, p=0.04, N N=2$, and $t=10^{7}$ iteration. (a) Distribution $P(F)$ of the fitness $F$ in the critical state (right curve) with the distribution of minimum fitness (left curve). (b) The distribution $P(D)$ of the distances $D$ between subsequent mutations in log-log plot. (c) Punctuated equilibrium behavior, activity vs time in a local segment of twenty consecutive species. (d) Distribution $P(S)$ of the avalanche size $S$ in the critical state in a log-log plot.

Figure 5 A system of size $N=4096, p=0.04, N N=2$, weight $=0.9$ and $t=10^{7}$ iteration. (a) Distribution $P(F)$ of the fitness $F$ in the critical state (right curve) with the distribution of minimum fitness (left curve). (b) The distribution $P(D)$ of the distances $D$ between subsequent mutations in log-log plot. (c) Punctuated equilibrium behavior, activity vs time in a local segment of twenty consecutive species. (d) Distribution $P(S)$ of the avalanche size $S$ in the critical state in a log-log plot.

Figure 6 A system of size $N=1000, p=0.04, N N=2$, weight $=0.9$ and $t=10^{7}$ iteration. (a) Distribution $P(F)$ of the fitness $F$ in the critical state (right curve) with the distribution of minimum fitness (left curve). (b) The distribution $P(D)$ of the distances $D$ between subsequent mutations in log-log plot. (c) Punctuated equilibrium behavior, activity vs time in a local segment of twenty consecutive species. (d) Distribution $P(S)$ of the avalanche size $S$ in the critical state in a log-log plot. 

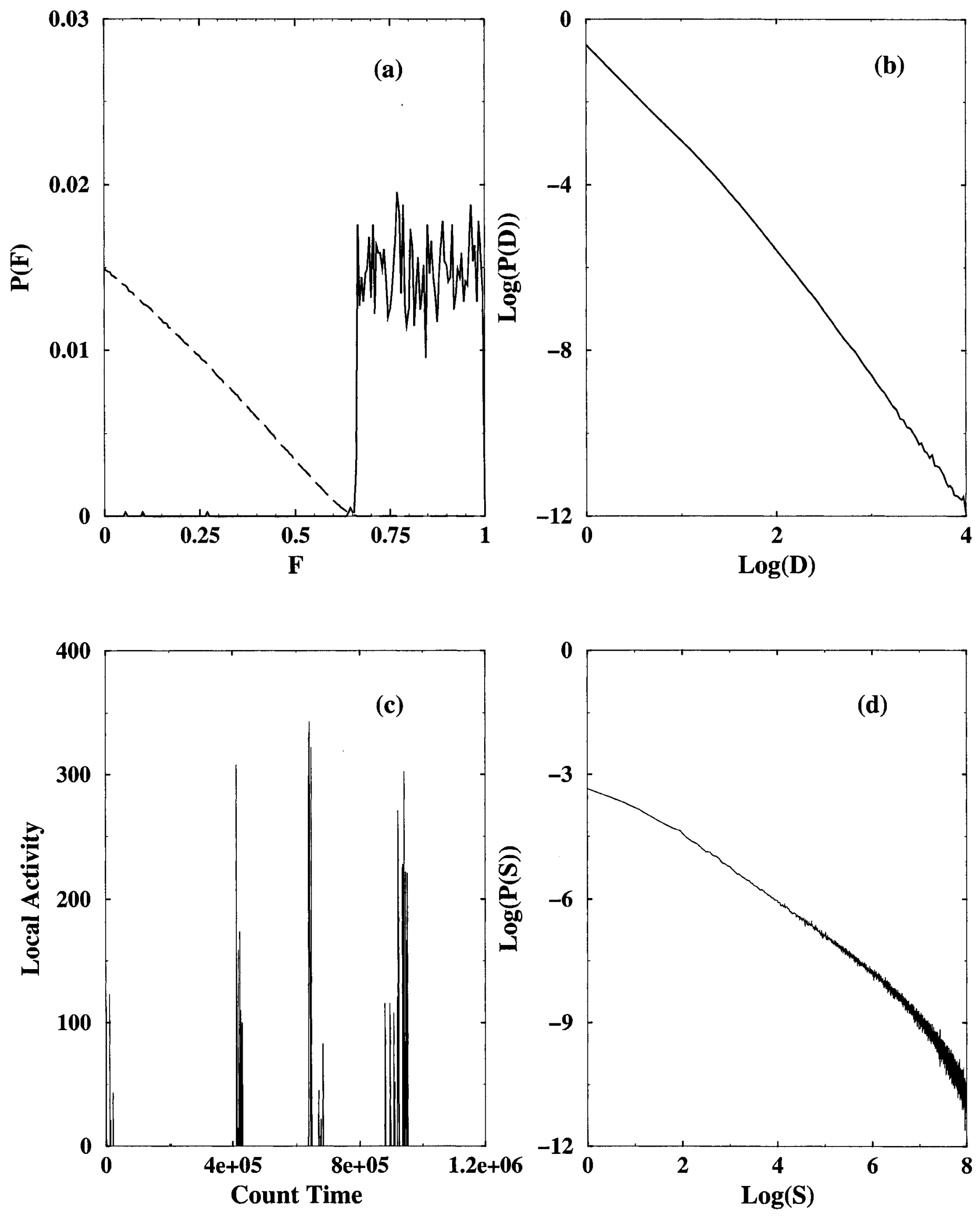

Figure (1) 

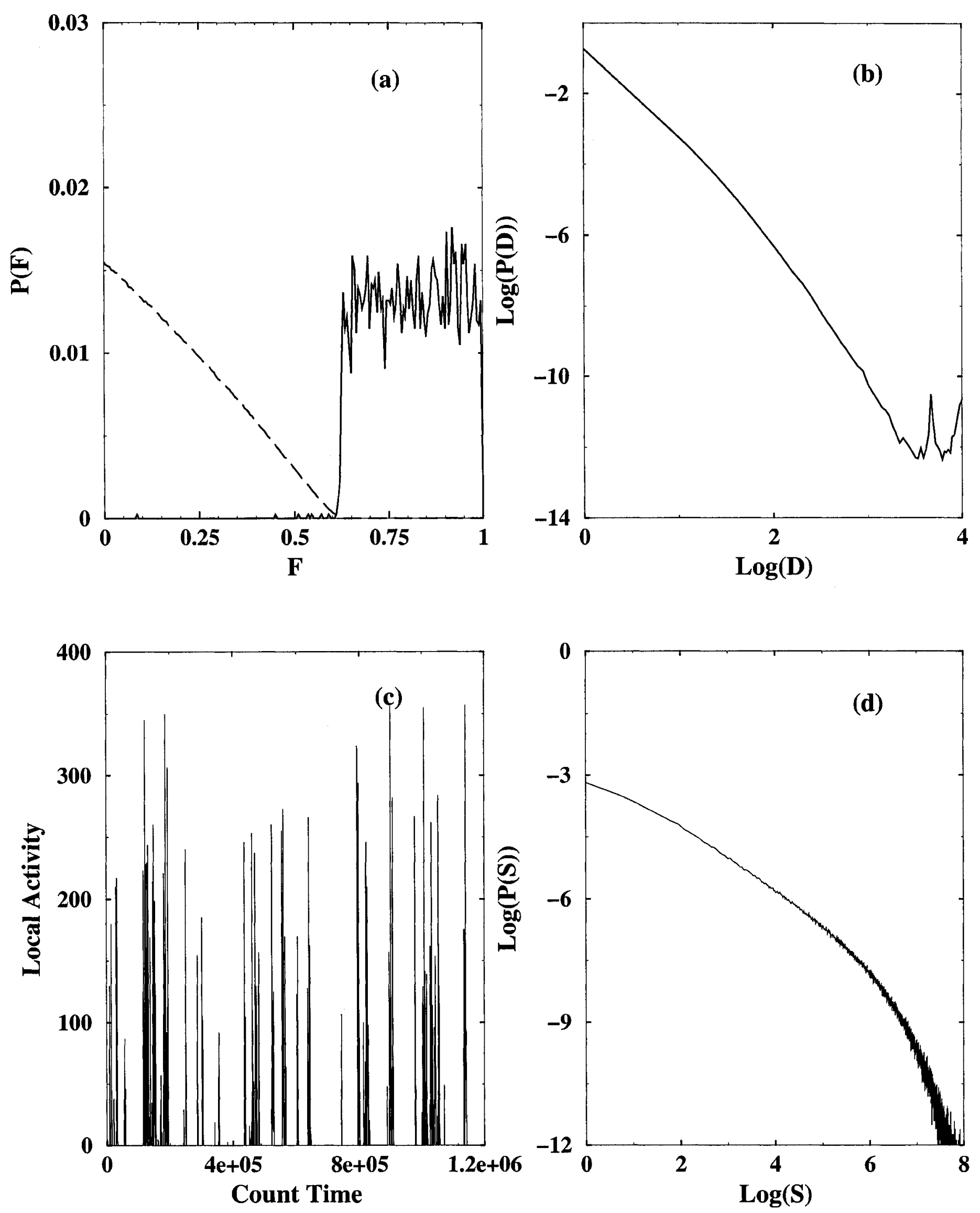

Figure (2) 

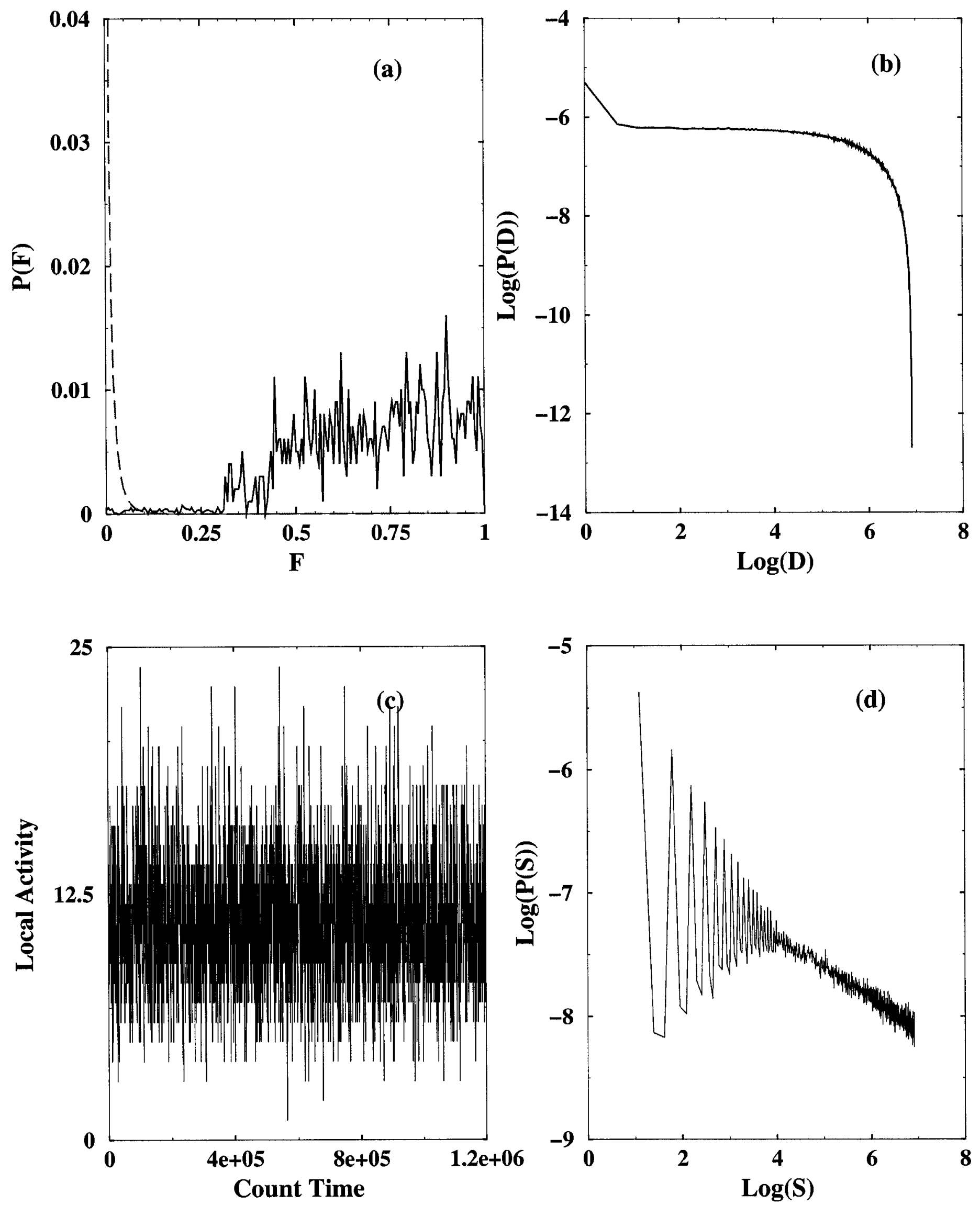

Figure (3) 

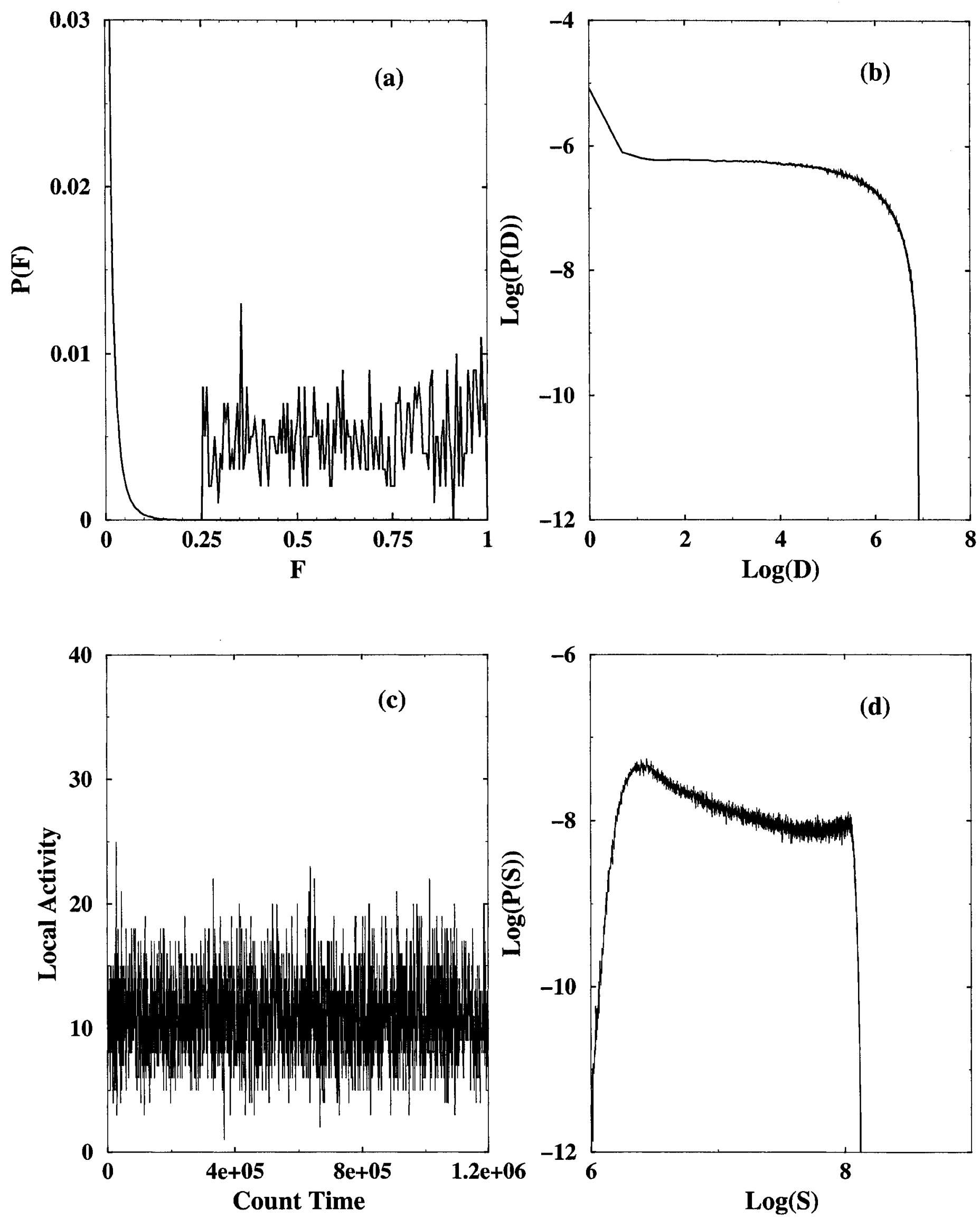

Figure (4) 

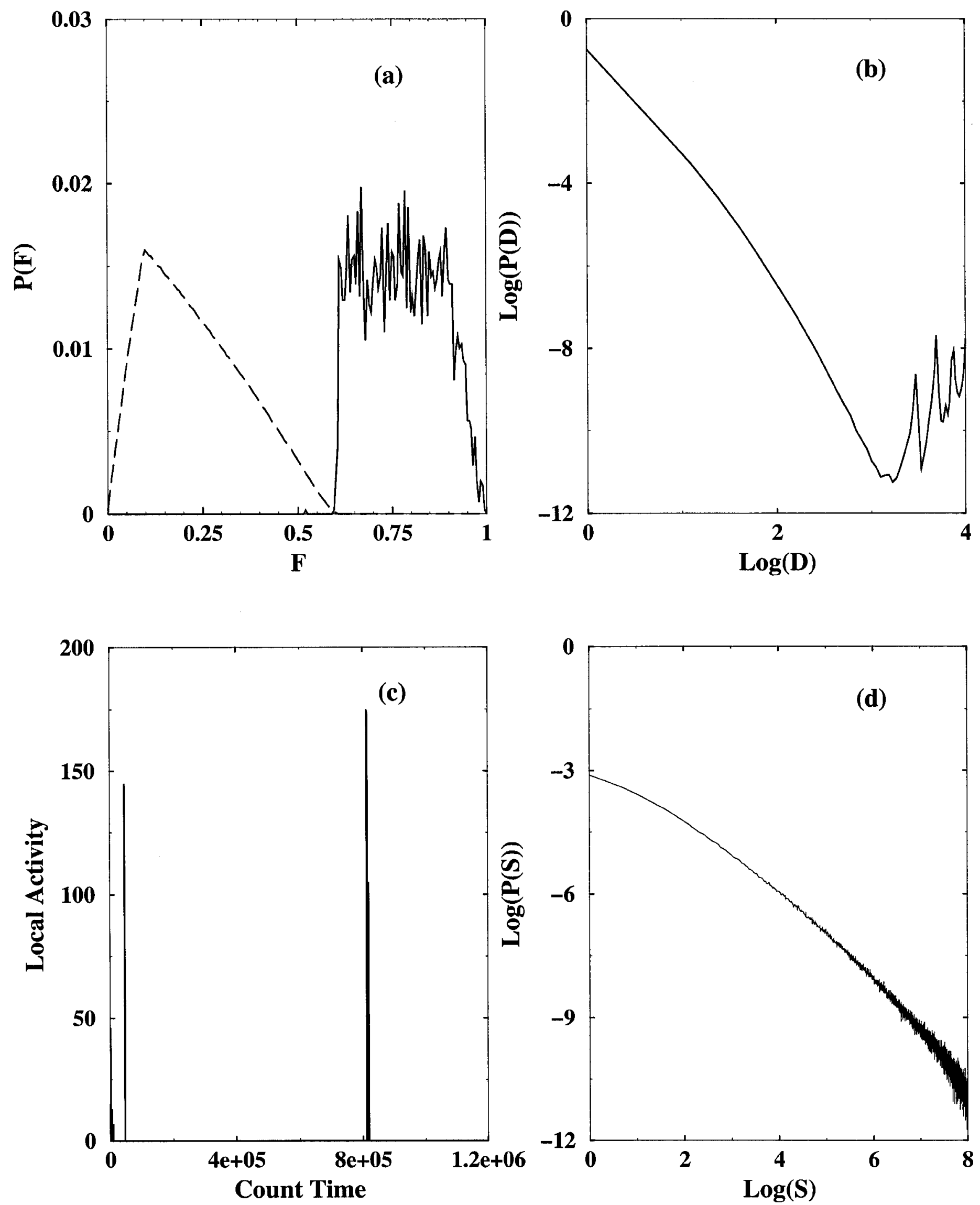

Figure (5) 

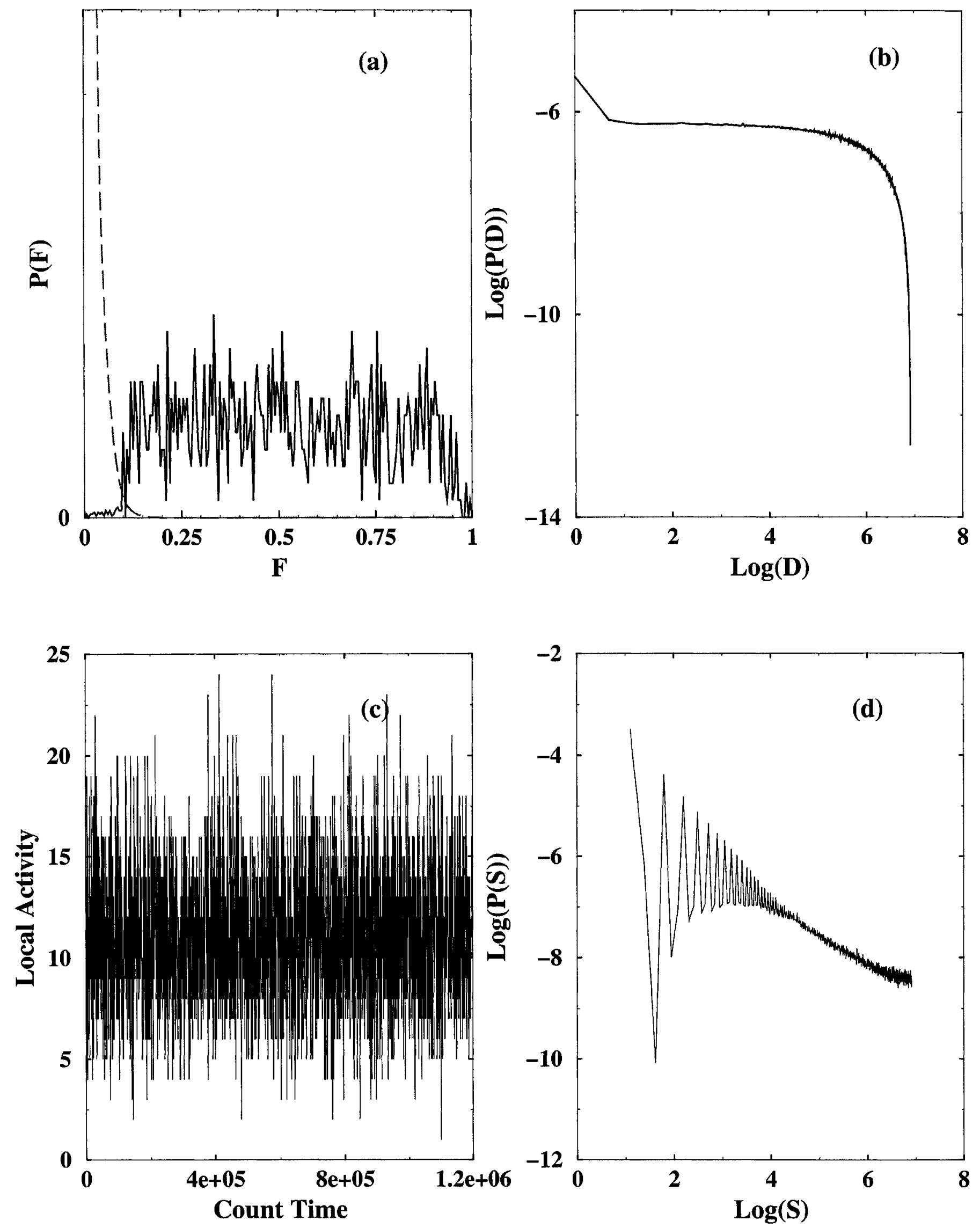

Figure (6) 